\title{
Comparison between intracranial collaterals and cerebral hemodynamics in patients with extracranial carotid stenosis versus combined extra- and intracranial stenosis using transcranial duplex
}

\author{
Sara Hamdy, Hani Mohamed Amin Aref, Ramez Reda and Ahmed ElSadek*
}

\begin{abstract}
Background: In acute ischemic stroke, collateral circulation plays an important role in maintaining blood flow to the tissue that is at risk of progressing into ischemia, and in increasing the successful recanalization rate without hemorrhagic transformation.

Objectives: To compare between collaterals in patients with extracranial carotid stenosis alone and in patients having both extracranial and intracranial carotid stenosis and therefore the difference in prognosis between them.

Materials and methods: A total of 30 patients of acute ischemic stroke were enrolled. The extracranial part of the carotid artery was assessed with color-coded duplex. Transcranial color-coded duplex was done to assess cerebral hemodynamics and collaterals to differentiate between extracranial significant carotid stenosis alone versus combined extra- and intracranial significant carotid stenosis.

Results: Out of 15 patients with significant extracranial stenosis, 3 of them obtained reversed flow in the ophthalmic artery and 3 patients of them showed change of flow in the anterior cerebral artery(ACA), whereas the other 15 patients of combined stenosis did not show any reversed blood flow in the same arteries which considered nonsignificant. The residual deficit of patients with extracranial stenosis alone was better when compared to that of the other group.

Conclusion: Our study concluded that transcranial duplex provides useful information about cerebral reserve and status of collaterals which show nonsignificant difference between two studied groups regarding intracranial collaterals. Furthermore, patients with combined extra- and intracranial stenosis were found to have more deficit and worse prognosis than patients with internal carotid stenosis only.
\end{abstract}

Keywords: Carotid stenosis, Collaterals, Transcranial color-coded duplex

\section{Introduction}

Transcranial color-coded duplex (TCCD) is highly suited to evaluate collateral circulation in carotid and intracranial steno-occlusive disease on account of its widespread availability and unique capacity to study real-time hemodynamics. It can reliably assess collateral flow via the circle of Willis and reversed ophthalmic artery flow in proximal ICA occlusion or severe stenosis [1].

\footnotetext{
* Correspondence: ahmedelsadek_79@yahoo.com

Neurology and Psychiatry, Ainshams University, 19/1/42, Elrehab city, Cairo P.O:11841, Egypt
}

\section{Springer Open}

Extracranial internal carotid artery atherosclerotic occlusive disease is a common ischemic stroke mechanism. Medical therapy refers to the contemporary approach of antiplatelet therapy, blood pressure control, low-density lipoprotein reduction, and lifestyle modification to reduce stroke risk. Carotid revascularization with endarterectomy or angioplasty and stenting are established treatments for patients with symptomatic carotid stenosis $\geq 70 \%$ [2]. In acute ischemic stroke, collateral circulation plays an important role in maintaining blood flow to the tissue that is at risk of progressing into ischemia, 
and in increasing the successful recanalization rate without hemorrhagic transformation. It has been reported that well-developed collateral circulation is associated with smaller infarct volume and better long-term neurological outcome, and it disappears promptly once the effective recanalization is achieved. Contrary to the belief that collateral vessels develop over time in chronic stenotic condition, there exists a phenomenon that collateral circulation develops immediately in acute stenosis or occlusion of the arteries [3].

\section{Aim of this work}

The aim of this study is to compare between collaterals in patients with extracranial carotid stenosis alone and in patients having both extracranial and intracranial carotid stenosis and therefore the difference in prognosis between them.

\section{Subjects}

Thirty patients with acute ischemic stroke were recruited in this prospective cohort study admitted in a stroke unit at the department of neurology of Ain Shams University hospitals.

\section{Inclusion criteria and subject selection}

Patients with clinical presentation of acute ischemic cerebrovascular stroke in the territory of anterior circulation with measurable deficit and able to give to give informed consent before enrollment in the study are divided into two groups: the first group includes 15 patients with high-grade extracranial stenosis (more than 70\%) detected by carotid duplex. The second group includes another 15 patients with both extracranial carotid stenosis detected by carotid duplex and intracranial stenosis detected by magnetic resonance angiography (MRA) (any intracranial stenosis whether middle cerebral artery (MCA) or ACA or posterior cerebral artery (PCA)).

\section{Exclusion criteria}

Patients were excluded from enrollment if they have nonsignificant carotid stenosis, clinical diagnosis did not correlate with acute stroke, or there was an evidence of intracranial hemorrhage.

\section{Methods}

A total of 30 patients of acute ischemic stroke were enrolled. All patients had detailed neurologic history and examination. The patient's neurological status was assessed via National Institute of Health and Stroke Scale (NIHSS) [4] and classified as follows: 1-4 mild stroke, 5-15 moderate stroke, and 16-20 moderate to severe stroke [5]. The modified Rankin Scale (mRS) was measured twice, first upon patients' discharge from the stroke unit and the second time 3 months later [6]. All patients had magnetic resonance imaging (MRI) of the brain (diffusion weighted, Flair, T1, T2, and T2* images) and magnetic resonance angiography (MRA) (Philips 1.5 Tesla, Germany). The extracranial part of the carotid artery was assessed with color-coded duplex using linear array probe $10-12 \mathrm{MHz}$ (Esaote mylab 5, Italy). Transcranial color-coded duplex used the same ultrasound system with phased array probe $2-4 \mathrm{MHz}$ to assess cerebral hemodynamics and collaterals. Hemodynamics were assessed by measuring peak systolic velocity, end diastolic velocity, and pulsitility index in the ipsilateral middle cerebral artery (MCA). Also, flow direction of the blood was assessed in both anterior cerebral arteries (ACA), posterior communicating artery and ophthalmic artery.

\section{Ethical consideration}

The study was approved by Ain-Shams University Ethical Committee. Written informed consent was obtained from the patients participating in the study, or their first degree relatives if the patient was unable to provide consent due to weakness or disturbed conscious level, after informing them about the study rationale and their right to withdraw from the study at any time without any consequences.

\section{Statistical analysis}

Statistical analysis was conducted using SPSS $\times 7$ (SPSS, IBM, Chicago, IL, USA). $P$ values were considered significant if $<0.05$. Descriptive statistics: mean, standard deviation $( \pm S D)$, and range were calculated for parametric numerical data, while median and range $(R)$ for nonparametric numerical data. Frequency and percentage were calculated for non-numerical data. Analytical statistics: Student $T$ test was used to assess the statistical significance of the difference between the two study group means. Correlation analysis was conducted to assess the strength of the association between two quantitative variables. The correlation coefficient denoted symbolically by " $r$ " defines the strength and direction of the linear relationship between two variables. Chi-square test was used to examine the relationship between two qualitative variables.

\section{Results \\ Demographic data}

Those 30 patients with acute ischemic cerebrovascular stroke include 17 males (56.67\%) and 13 females (43.33\%) - in those 15 patients with extracranial stenosis, their ages vary from 30 to 80 years, with mean $59.667 \pm$ 14.56 , and in those with both intracranial and extracranial stenosis, their ages vary from 57 to 74 years, with mean $66.133 \pm 5.303$. 
Comparison between patients with extracranial stenosis and patients with both intracranial and extracranial stenosis as regards anterior cerebral artery, posterior communicating artery flow

In those 15 patients with significant extracranial stenosis, $3(20 \%)$ of them showed change of flow in ACA, showing that contralateral ACA was the donor artery, whereas the other 15 patients of both extracranial and intracranial stenosis did not show any reversed blood flow in their ACAs or posterior communicating artery (Table 1).

Comparison between patients with extracranial stenosis and patients with both intracranial and extracranial stenosis as regards ophthalmic artery flow In those 15 patients with significant extracranial stenosis, $3(20 \%)$ of them obtained reversed flow in the ophthalmic artery, whereas the other 15 patients of both extracranial and intracranial stenosis did not show any reversed blood flow in their ophthalmic arteries (Table 2).

No reversed flow was noted in the posterior communicating artery of all patients.

\section{Comparison between patients with extracranial stenosis} and both intracranial and extracranial stenosis as regards PSV and EDV

Regarding those 30 patients with acute stroke, cerebral hemodynamics were assessed by transcranial Doppler by the assessment of both PSV and EDV in ipsilateral MCAs; in the 15 patients of extracranial stenosis, only one patient $(6.67 \%)$ showed increased PSV over $155 \mathrm{~cm} /$ $\mathrm{s}$ [7], with mean $59.38 \pm 22.25$, blunted flow (tardus paravus) in proximal M1 segment of 26 patients and distal M1 in one patient.

While in 15 patients of both intracranial and extracranial stenosis, also only one patient $(6.67 \%)$ showed increased PSV in MCA over $160 \mathrm{~cm} / \mathrm{s}$, with mean $68.133 \pm 26.963$ (Fig. 1), blunted flow (tardus paravus) in proximal M1 segment of 10 patients and distal M1 in one patient.
Comparison between patients with extracranial stenosis and patients with both intracranial and extracranial stenosis as regards NIHSS

Those 15 patients with extracranial stenosis only showed less NIHSS (less deficit) with mean $8.333 \pm 2.795$ than those 15 patients with both intracranial and extracranial stenosis who showed higher NIHSS (more deficit) with mean $12.733 \pm 2.631$ (Table 3).

\section{Comparison between patients with extracranial stenosis and patients with both intracranial and extracranial stenosis as regards mRS upon discharge and after 3 months follow-up}

Those 15 patients with extracranial stenosis showed less mRS both upon discharge with mean $2.067 \pm 1.033$ and after 3 months with mean $1.200 \pm 0.941$ than those 15 patients with both intracranial and extracranial stenosis both upon discharge with mean $2.800 \pm 0.862$ and after 3 months with mean $2.933 \pm 1.534$. Consequently, the first group with only extracranial stenosis showed more significant improvement than the second group (Fig. 2). Three patients with active collaterals show less mRS after 3 months with mean 0.67 in comparison to discharge mRS with mean 1.67 .

\section{Discussion}

Use of transcranial Doppler ultrasound includes detection of site/degree of stenosis/occlusion of cerebral vasculature, assessment of recanalization following occlusion (with/ without thrombolytic treatment), and assessment of collateral flow in intracranial vasculature in cases of critical carotid artery stenosis (extracranial) [8].

Our main aim in this study is to assess collateral circulation in significant carotid stenosis with intracranial stenotic vessels by using transcranial color-coded duplex to determine whether they are worse or not than collaterals in cases of internal carotid stenosis alone, and consequently if the prognosis of those patients is worse or not. Collateral flow patterns are important risk factors for brain ischemia in the presence of internal carotid artery (ICA) stenosis or occlusion. So, contralateral anterior cerebral artery flow and ophthalmic artery (OA) flow

Table 1 Difference in ACA flow in both groups

\begin{tabular}{|c|c|c|c|c|c|c|c|c|}
\hline \multirow{3}{*}{$\begin{array}{l}\text { Ipsilateral } \\
\text { ACA }\end{array}$} & \multicolumn{6}{|l|}{ MRA } & \multirow{2}{*}{\multicolumn{2}{|c|}{ Chi-square }} \\
\hline & \multicolumn{2}{|c|}{ Extracranial stenosis } & \multicolumn{2}{|c|}{ Intracranial and extracranial stenosis } & \multicolumn{2}{|c|}{ Total } & & \\
\hline & $N$ & $\%$ & $s$ & $\%$ & $N$ & $\%$ & $\overline{x^{2}}$ & $P$ value \\
\hline Normal & 12 & 80.00 & 15 & 100.00 & 27 & 90.00 & 3.333 & 0.068 \\
\hline Reverse & 3 & 20.00 & 0 & 0.00 & 3 & 10.00 & & \\
\hline Total & 15 & 100.00 & 15 & 100.00 & 30 & 100.00 & & \\
\hline
\end{tabular}


Table 2 Difference in ophthalmic artery flow in both groups

\begin{tabular}{|c|c|c|c|c|c|c|c|c|}
\hline \multirow{3}{*}{$\begin{array}{l}\text { Ophthalmic } \\
\text { artery }\end{array}$} & \multicolumn{6}{|l|}{ MRA } & \multirow{2}{*}{\multicolumn{2}{|c|}{ Chi-square }} \\
\hline & \multicolumn{2}{|c|}{ Extracranial stenosis } & \multicolumn{2}{|c|}{ Intracranial and extracranial stenosis } & \multicolumn{2}{|c|}{ Total } & & \\
\hline & $N$ & $\%$ & N & $\%$ & $\bar{N}$ & $\%$ & $x^{2}$ & $P$ value \\
\hline Reverse & 3 & 20.00 & 0 & 0.00 & 3 & 10.00 & 3.333 & 0.068 \\
\hline Forward & 12 & 80.00 & 15 & 100.00 & 27 & 90.00 & & \\
\hline Total & 15 & 100.00 & 15 & 100.00 & 30 & 100.00 & & \\
\hline
\end{tabular}

reversal are studied by transcranial Doppler sonography, as they are important markers for high-grade ICA stenosis or occlusion and in detection of prognosis [9].

Our study is considered pioneer in comparing hemodynamics in cerebral collaterals between these two groups of patients, but results revealed that there is no difference statistically between them, may be due to small sample size. Our study shows that in 15 patients with significant extracranial stenosis, 3 (20\%) of them obtained reversed flow in the ophthalmic artery and 3 patients of them (20\%) showed change of flow in ACA (showing that contralateral ACA was the donor artery) whereas the other 15 patients of both extracranial and intracranial stenosis did not show any reversed blood flow in their ophthalmic arteries or any reversed blood flow in their ACAs; however, this was statistically not significant probably due to small sample size. These findings were somewhat different from the study done by Yueh-Feng Sung and his coworkers [10]; they assessed the clinical implications of reversed ophthalmic artery flow (ROAF) for stroke risk and outcomes in subjects with unilateral severe cervical carotid stenosis/occlusion. They investigated 128 subjects (101 with acute stroke and 27 without), selected from a large hospital patients base $(n=14,701)$, identified with unilateral high-grade cervical carotid stenosis/occlusion by using duplex ultrasonography and brain magnetic resonance imaging. All clinical characteristics were compared for stroke risk between acute stroke and nonstroke groups. Patients with acute stroke were divided into four subgroups according to ophthalmic artery flow direction and intracranial stenosis severity, and stroke outcomes were evaluated. The acute stroke group had significantly higher percentages of $\operatorname{ROAF}(52.5 \%, p=0.003)$, carotid occlusion (33.7\%, $p=0.046)$, and severe intracranial stenosis $(74.3 \%, p<0.001)$. However, multivariate analysis demonstrated that intracranial stenosis was the only significant risk factor (odds ratio $=10.38$; 95\% confidence interval $=3.64-29.65 ; p<0.001$ ). Analysis of functional outcomes among the four subgroups of patients with stroke showed significant trends $(p=0.018$ to 0.001$)$ for better stroke outcomes from ROAF and mild or no intracranial stenosis. ROAF improved $10-20 \%$ stroke outcomes, as compared to forward ophthalmic artery flow, among the patients with stroke and the same degree of

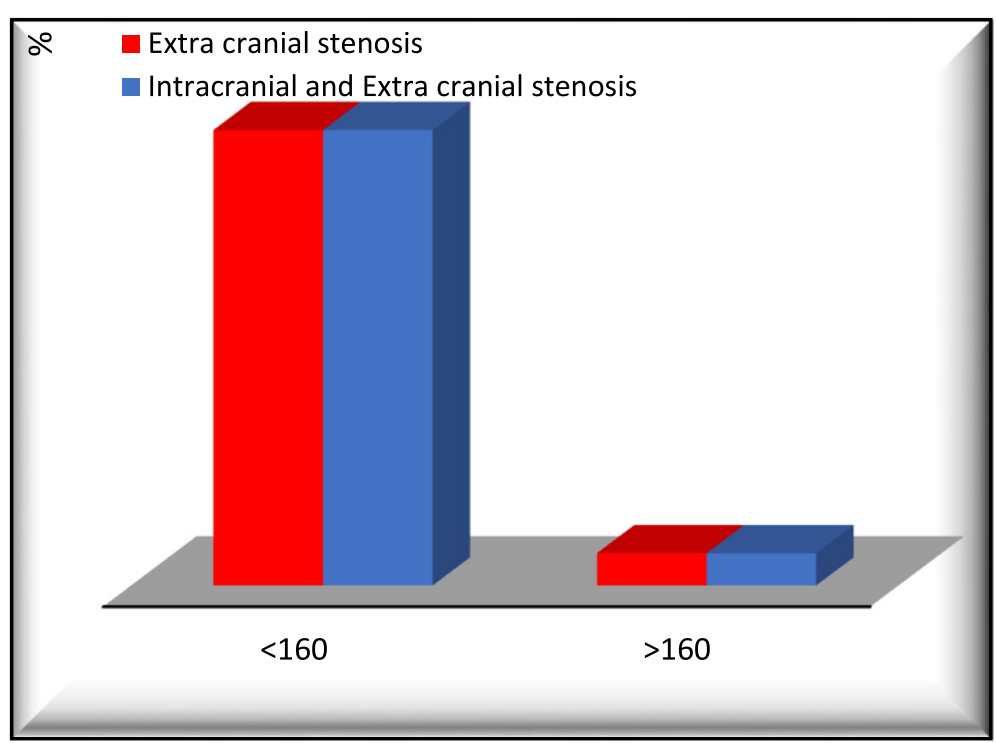

Fig. 1 PSV in both groups. Regarding those 30 patients with acute stroke, cerebral hemodynamics were assessed by transcranial Doppler by the assessment of both PSV and EDV in ipsilateral MCAs; in the 15 patients with extracranial stenosis, only one patient (6.67\%) showed increased PSV over $160 \mathrm{~cm} / \mathrm{s}$, with mean $59.38 \pm 22.25$, while in 15 patients of both intracranial and extracranial stenosis, also only one patient (6.67\%) showed increased PSV in MCA over $160 \mathrm{~cm} / \mathrm{s}$ with mean $68.133 \pm 26.963$ 
Table 3 Difference in NIHSS between the two groups

\begin{tabular}{|c|c|c|c|c|c|c|c|c|}
\hline \multirow{3}{*}{$\begin{array}{l}\mathrm{NIH} \\
\text { Range }\end{array}$} & \multicolumn{6}{|l|}{ MRA } & \multicolumn{2}{|l|}{$T$ test } \\
\hline & \multicolumn{3}{|c|}{ Extracranial stenosis } & \multicolumn{3}{|c|}{ Intracranial and extracranial stenosis } & \multirow{2}{*}{$\frac{T}{-4.440}$} & \multirow{2}{*}{$\frac{P \text { value }}{<0.001}$} \\
\hline & 2 & - & 12 & 8 & - & 18 & & \\
\hline Mean \pm SD & 8.333 & \pm & 2.795 & 12.733 & \pm & 2.631 & & \\
\hline
\end{tabular}

* Highly significant

severities of intracranial stenosis. So, this study stated that the patients with acute stroke and severe unilateral cervical carotid stenosis/occlusion significantly have high incidence of intracranial stenosis and reversal of flow in the ophthalmic artery.

In our study, the residual deficit regarding the two groups was compared and we found that those 15 patients with only extracranial stenosis were better with less NIH than those with combined intracranial and extracranial stenosis. Furthermore, we also found that prognosis of patients with only extracranial stenosis after 3 months was better than that of patients with both extracranial and intracranial stenosis. This was found also in a study done by Tsai and his colleagues [5], who stated that patients with unilateral high-grade cervical carotid stenosis/occlusion in combination with intracranial stenosis appear to be a significant risk factor for poor functional outcome. In comparison to the previous studies, our study is considered pioneer in comparing hemodynamics in cerebral collaterals between these two groups of patients, but results revealed that there is no difference statistically between them, may be due to small sample size.
However, some limitations could be noted in this study as we did not take a third group to study cerebral hemodynamics in intracranial stenosis alone in order to compare them with the cerebral hemodynamics found in the two groups already taken in this study. Also, the number of candidates studied was considered limited which may be the cause of some nonsignificant results.

\section{Conclusion}

Transcranial color-coded duplex is safe, accurate, inexpensive, non-invasive, and a bedside technique providing useful information on intracerebral vasculature. It was used in this study to compare cerebral collaterals (direction of flow in ophthalmic arteries and anterior cerebral arteries) in cases with internal carotid stenosis alone or cases with both internal carotid and intracranial stenosis together which show nonsignificant difference. Furthermore, patients with both internal carotid and intracranial stenosis were found to have more deficit and worse prognosis after 3 months than patients with internal carotid stenosis only.

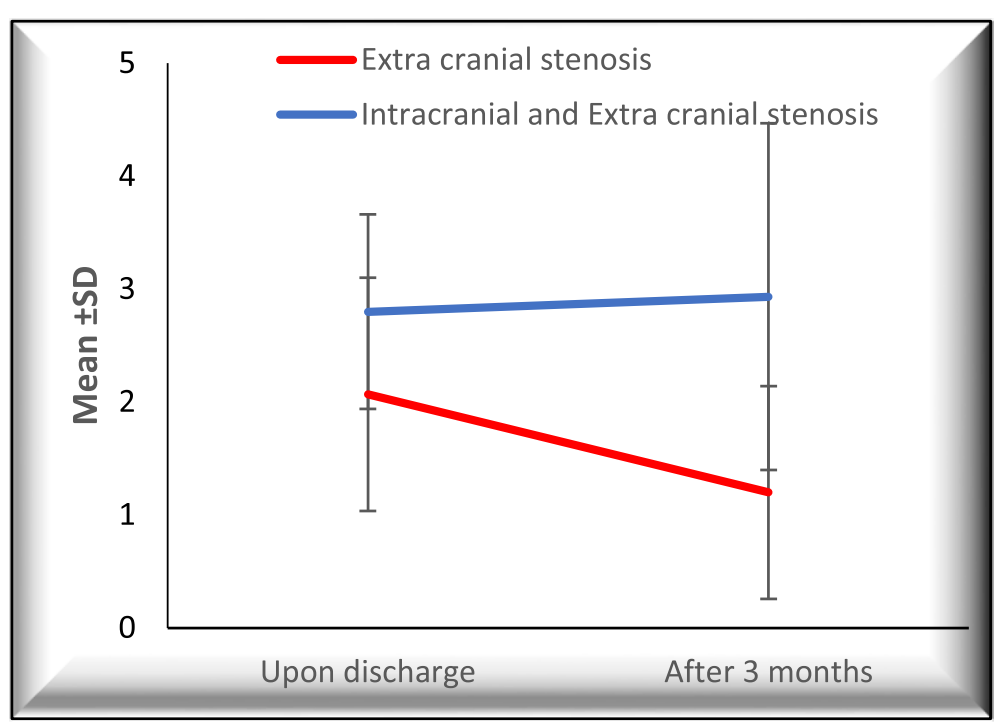

Fig. 2 Difference in mRS in both groups. Those 15 patients with extracranial stenosis showed less mRS both upon discharge with mean $2.067 \pm$ 1.033 and after 3 months with mean $1.200 \pm 0.941$ than those 15 patients with both intracranial and extracranial stenosis both upon discharge with mean $2.800 \pm 0.862$ and after 3 months with mean $2.933 \pm 1.534$. Consequently, the first group with only extracranial stenosis showed more significant improvement than the second group 


\section{Abbreviations}

ACA: Anterior cerebral artery; MCA: Middle cerebral artery; MRA: Magnetic resonance angiography; mRS: Modified Rankin Scale; NIHSS: National Institute of Health and Stroke Scale; PCA: Posterior cerebral artery; TCCD: Transcranial color-coded duplex

\section{Acknowledgements}

not applicable

\section{Funding}

not applicable

\section{Availability of data and materials}

Dataset is available as master sheet in Excel format and publicly available in Neurology department, Ain Shams University.

\section{Authors' contributions}

SH participated in the design of the study and performed the statistical analysis (ES). HMAA conceived of the study and participated in its design and coordination and helped to draft the manuscript (FG). RR conceived of the study and participated in its design and coordination and helped to draft the manuscript ( $F G$ ). AE conceived of the study and participated in its design and coordination and helped to draft the manuscript (FG). All authors read and approved the final manuscript.

\section{Ethics approval and consent to participate}

The study was approved by AIN-SHAMS UNIVERSITY ETHICAL COMMITTEE. Written informed consent was obtained from the patients participating in the study, or their first degree relatives if the patient was unable to provide consent due to weakness or disturbed conscious level, after informing them about the study rationale and their right to withdraw from the study at any time without any consequences.

\section{Consent for publication}

not applicable

\section{Competing interests}

The authors declare that they have no competing interests.

\section{Publisher's Note}

Springer Nature remains neutral with regard to jurisdictional claims in published maps and institutional affiliations.

Received: 26 May 2018 Accepted: 15 January 2019

Published online: 11 February 2019

\section{References}

1. Alexandrov AV, Sloan MA, Wong LK, Douville C, Razumovsky AY, Koroshetz WJ, Kaps M, Tegeler CH. American Society of Neuroimaging Practice Guidelines Committee. Practice standards for transcranial Doppler ultrasound: part I - test performance. J Neuroimaging. 2007;17:11-8.

2. Barrett KM, Brott TG. Stroke caused by extracranial disease. Circ Res. 2017; 120(3):496-501.

3. Iwasawa E, Ichijo M, Ishibashi S, Yokota T. Acute development of collateral circulation and therapeutic prospects in ischemic stroke. Neural Regen Res. 2016;11(3):368-71.

4. Hage V. The NIH stroke scale: a window into neurological status. NurseCom Nursing Spectrum (Greater Chicago) [serial online]. 2011:24(15):44-9.

5. Tsai CL, Lee JT, Cheng CA, Liu MT, Chen CY, Hu HH, Peng GS. Reversal of ophthalmic artery flow as a predictor of intracranial hemodynamic compromise: implication for prognosis of severe carotid stenosis. Eur J Neurol. 2013;20(3):564-70.

6. Saver JL, Filip B, Hamilton S, et al. Improving the reliability of stroke disability grading in clinical trials and clinical practice: the Rankin Focused Assessment (RFA). Stroke. 2010;41(5):992-5.

7. Baumgartner RW, Mattle HP, Schroth G. Assessment of $\geq 50 \%$ and $<50 \%$ intracranial stenoses by transcranial color-coded duplex sonography. Stroke. 2018;30:87-92.

8. Saqqur M, Zygun D, Demchuk A. Role of transcranial Doppler in neurocritical care. Crit Care Med. 2007;35(5 Suppl):S216-23.
9. Reynolds PS, Greenberg JP, Lien LM, Meads DC, Myers LG, Tegeler CH. Significant ICA stenosis. J Neuroimaging. 2002;12(1):5-8.

10. Sung Y-F, Tsai C-L, Lee J-T, Chu C-M, Hsu C-H, Lin C-C, Peng G-S. Reversal of ophthalmic artery flow and stroke outcomes in Asian patients with acute ischemic stroke and unilateral severe cervical carotid stenosis. PLoS ONE. 2013;8(12):e80675.

\section{Submit your manuscript to a SpringerOpen ${ }^{\circ}$ journal and benefit from:}

- Convenient online submission

- Rigorous peer review

- Open access: articles freely available online

- High visibility within the field

- Retaining the copyright to your article

Submit your next manuscript at $\boldsymbol{\nabla}$ springeropen.com 